\title{
Challenges and Issues for Routing in Mobile Ad Hoc Network - Review
}

\author{
Nikita Jaiswal \\ Computer science and Engineering \\ Shree vaishnav Institute of technology and \\ Science Indore \\ (M.P), India
}

\begin{abstract}
MANETs Routing is a one of the dynamically and ambitious task and has received a great amount of awareness from researchers around the globe. To overcome this problem, a various number of routing classes have been introduced and the number is still spiraling day by day. It is quite hard to decide which protocols or routing classes may do well under an amount of disparate network scenarios such as network volume and network topology etc. In this paper, we present a summary of a large range of the existing routing classes with a particular focus on their uniqueness and their functionality. Also, the intuition is provided based on the routing functionality and information is used to build routing decisions. The presentation of all the routing protocols or classes is also discussed. Further this study will abetment the researchers to get a summary of the existing classes and advice which protocols may execute better with respect to varying between network scenarios.
\end{abstract}

\section{Keywords}

MANET, Routing protocols, comparison of Routing protocols

\section{INTRODUCTION}

Mobile Ad Hoc Networks (MANETs) have reaped an increasing significance. Ad Hoc networking is needed in many applications such as military and battlefield operations, virtual classrooms or conference rooms, and salvage operations in natural disasters. These kinds of applications require a network regardless of any infrastructure, and this is the idea behind MANETs which can be considered as malleable networks and suitable for such applications. MANETs are typically characterized by high mobility and frequent link failures that result in low throughput and high end-to-end delay. The increasing use of MANETs for ferry multimedia applications such as voice, video and data, leads to the need to provide QoS support.

In mobile networks, node mobility makes the network topology change intermittently, which is rare in wired networks. Mobile networks have a high error rate, bandwidth limitations and power restraint. Due to the impacts from transmission power, receiver sensitivity, noise, fading and interference, wireless link capacity continually varies. Wireless networks can be deployed quickly and easily, and users stay connected to the network while they are moving everywhere. Also, they play an important role in both civilian and military fields. We have seen great developments in Wireless networks framework, availability of wireless applications, and proliferation of Wireless devices everywhere such as laptops, PDAs, and cell phones.

\author{
Rupali Bhartiya \\ Asst. prof. Computer science and Engineering \\ Shree vaishnav Institute of technology and \\ Science Indore \\ (M.P), India
}

\section{CHARACTERISTICS OF MANETS}

The design of routing protocol for MANET is delicate because of numerous network limitations. MANET experience from the restrictions of several network resources, for examples, energy, bandwidth, processor, and storage, the aim of threat in sensor networks involves the following main aspects.

Dynamic topology:- Nodes are free to move arbitrarily in any direction thus the topology of the network change unpredictably.

Limited Bandwidth:- The bandwidth available for wireless networks is generally low than that of wired networks. The throughput of these networks is generally low due various noises, fading effects.

Energy constrained operation:- The nodes are portable devices and are dependent on batteries. This is the most important design consideration of the MANET

\section{SECURITY}

Wireless networks are more prone to hazard than wired networks. The increased possibility of various security attacks like eavesdropping, denial of Service should be handled carefully. The performance of MANET depends on the routing protocol, battery utilization by the nodes. There is Various Quality of service parameters which affect the performance like a bandwidth delay, jitter, throughput etc. Due to dynamic topology routing is the major confront these networks because the bandwidth provided to the nodes at one point of time becomes unavailable if the nodes move from a appropriate position and go to other position. Moreover, routing affects the performance of these networks. Therefore efficient routing protocol needs to be developed to meet all these challenges. Routing protocol in MANET is coordination into three categories on the basis of route discovery reactive also called as the ondemand routing protocol, proactive also known as the table driven protocol and Hybrid protocol. Further classification of routing protocols is done on the basis of network organization as flat based, hierarchical based and location based. In flat based protocol all the nodes are equal i.e. they play the same role in the network. In hierarchical protocol different nodes play different roles i.e. in this different cluster heads are chosen among cluster members. In location-based protocol nodes rely on the location information and use this information for communication. 


\section{ROUTING PROTOCOLS FOR MANETS}

Routing is an action to move the information from source point to destination point within the network. During this process, at least, one intermediate node within the network is encountered. The routing notion basically involves, two activities: firstly, determining best possible routing paths and secondly, transferring the information throughout a network. Routing basically divided into two types: static routing and dynamic routing. Static routing refers to the routing policy being manual. In static routing maintains a routing table by the administrator. Dynamic routing mainly depends upon the state. Mobile ad hoc network present the dynamic routing [10]. These protocols can be divided into three classes ${ }^{\text {ee }}$ proactive class, reactive class and hybrid class as shown in figure 2.This classification of routing protocols are work according to their technique such as hop count, link state and QoS in route discovery. Hop count method, each node contains next hop information in its routing table, to the destination while link state routing protocols keep a routing table for absolute topology, which is built up by finding shortest path of link costs. QoS routing is the procedure of selecting the path to be used by the packets of a flow, based on its QoS requirements eg bandwidth, delay etc [4].

Ad Hoc on Demand Distance Vector Routing (AODV):AODV routing protocol described in [21] builds on the DSDV algorithm previously described. AODV is an improvement on DSDV because it typically minimizes the number of required broadcasts by creating routes on a demand basis, as opposed to maintaining a complete list of routes as in the DSDV algorithm. The authors of AODV classify it as a pure on demand route acquisition system, since nodes that are not on a selected path do not maintain routing information or participate in routing table exchanges [21]. When a source node desires to send a message to some destination node and does not already have a valid route to that destination, it initiates a path discovery process to locate the other node. It broadcasts a route request (RREQ) packet to its neighbors, which then forward the request to their neighbors, and so on, until either the destination or an intermediate node with a - fresh enough\| route to the destination is located.

Temporally-Ordered Routing Algorithm (TORA):- TORA routing class. It was developed by Park and Corson. Temporarily ordered routing algorithm (TORA) is the class that truly adaptive, loop-free, discrete routing algorithm based on the concept of connection turnaround. It uses directed acyclic graphs (DAG) to explain the routes either upstream as well as downstream. However to give this feature, TORA wants synchronization of the nodes which restrictions the function of the protocol. TORA routing class is a moderately complex protocol but what makes it unique and important is its main characteristic of the broadcast of manage messages only around the point of the crash when a link stoppage occurs. In the evaluation, all the other protocols require to re-initiate a route detection when a link not succeed but TORA would be able to patch itself up around the point of failure. This characteristic allows TORA to level up to bigger networks, but it has a higher overhead for smaller networks. TORA occupy four key operations: creating, preserving, removing and optimizing routes. Since every node must have a height, any node which does not have a height is calculated as a removed node and its height is considered as null.
Sometimes the nodes are given new heights to get better the linking structure. This purpose is called optimization of routes.

Dynamic Source Routing (DSR):- DSR is a widely used reactive (on-demand) routing protocol which is designed particularly for the mobile ad-hoc networks. DSR permits the network to run without any existing network infrastructure and thus the network becomes as a selforganized and self- configured network. This protocol maintains an on-demand approach and hence extinguishes the periodic table-update messages needed in the tabledriven approach [22]. Consequently, it is able to prevent the control packets from consuming much bandwidth. Like other on-demand routing protocols, DSR does not provide the transmission of any periodic hello packet (beacon), which is essential for informing its presence to other nodes. Instead, during the route construction phase, it establishes the route by flooding a Route Request packet in the network. Each Route Request packet holds a sequence number which is generated by all the nodes through which the packet is flooded.

Destination Sequenced Distance Vector (DSDV):- DSDV is a proactive routing protocol which maintains routes regardless of their usage. It is based on the Bellman-Ford routing algorithm, which can become unacceptable in mobile ad hoc networks because of its long convergence time. Numerous extensions or modifications to DSDV have been proposed to improve its performance such as [9] DSDV is a distance vector routing protocol and it solves the major problem associated with the Distance Vector routing of wired networks (i.e., Count-to-infinity), by using destination sequence numbers. Also, at all times, the DSDV protocol guarantees loop-free paths to each destination.

Using DSDV, each mobile node maintains a routing table that lists one route for each destination. Each routing table entry consists of the destination node, the first hop towards the destination, the metric (number of hops to reach the destination), and the sequence number which is originally generated by the destination node. Sequence numbers are used to distinguish the new routes from the stale routes. The routing table is used to transmit packets between the nodes of the network.

Optimized Link State Routing (OLSR):- OLSR is the proactive class that employs a capable link state packet forwarding system called multipoint relaying [11, 12]. It optimizes the clean link state routing class. There are two ways to done Optimizations: by reducing the volume of the control packets and the Second way by tumbling the number of associations used for promoting the link state packets. As you know that each and every node preserves the topology information about the network by periodically replacing link-state communication among the other nodes. OLSR routing class is based on the following three mechanisms: neighbor sensing, capable flooding, and calculation of an optimum route using the variety of shortest-path algorithm. Neighbor sensing is the judgment of changes in the region of the node. Each node finds out the best route to every known target using this topology information and accumulates this information in a routing table. The shortest path algorithm is then used for calculating the most constructive path. Routes to every destination are directly available when data broadcast begins furthermore remaining suitable for a specific period of time till the information is ended. 
Wireless Routing Protocol (WRP):- WRP class, as planned by Garcia-Luna-Aceves [17], it is a table-based class related to DSDV class that take over the assets of BellmanFord Algorithm. The main aim is preserving routing information among a variety of nodes in the network about the shortest path to every target. Wireless routing protocols (WRP) is a loop-free routing class. WRP is a pathsearching algorithm with the exemption of shunning the count-to-infinity disaster by forcing each node to execute constancy checks of precursor information detailed by all its neighbors. Each and every node in the network implies a set of four tables to carry on more precise information. these are following tables Distance table (DT), Routing table (RT), Link-cost table (LCT), Message retransmission list (MRL) table. In that case of link breakdown between two nodes, the nodes launch bring up to date communications to their neighbors. WRP fit into the class of path searching algorithms with a significant exception. It counters the count-to-infinity difficulty by forcing each node to do consistency checks of precursor information statemented by all its neighbors. This removed looping circumstances and enables quicker route convergence when a link failure occurs.

\section{CONCLUSION}

In this article, we have presented and discussed the taxonomy of routing protocols in mobile ad hoc networks and provided comparisons between them. The protocols are divided into two main categories: (i) source-initiated (reactive or on-demand), (ii) table-driven (pro-active). For each of these classes, we reviewed and compared several representative protocols. While there are still many challenges facing Mobile ad hoc networks complementary to routing and security. Each routing protocol has unique features. Based on network environments, we have to choose the relevant routing protocol. The analysis of the different proposals has demonstrated that the inherent characteristics of ad hoc networks, such as lack of infrastructure and rapidly changing topologies, introduce additional difficulties to the already complicated dilemma of secure routing. The main differentiating factor between the protocols is the ways of finding and maintaining the routes between source-destination pairs. The comparison we have conferred between the routing protocols indicates that the design of a secure ad hoc routing protocol constitutes a challenging research dilemma against the existing security solutions. We hope that the taxonomy presented in this paper will be helpful and provide researchers a platform for choosing the right protocol for their work, at last we have provided the comprehensive characteristic features of all routing protocols and described which protocols may perform best in large networks. Almost all the protocols we discussed in this article have their own characteristic features and performance parameter combinations where they outperform their competitors, Still mobile ad hoc networks have posed an enormous challenge for the researchers due to fast changing topology and security attacks, and none of the protocols is fully secured and research is going on around the globe.

\section{REFERENCES}

[1] Sumathia K. and Priyadharshinib A.,\| energy optimization in manets using on demand routing protocoll elsevier, procedia computer science 47 (2015 ) $460-470$.

[2] Dhawan S. and Saroha V.,\| optimize the routing protocol (grp, olsr, dsr) using opnet \& its performance evaluationll, international journal of advances in engineering \& technology, vol. 6, issue3, pp. 1399 . 1408 .

[3] Menon V. and Prathap J., -Performance Analysis of Geographic Routing Protocols in Highly Mobile Ad Hoc Network\|, Journal of Theoretical and Applied Information Technology, $\quad$ Vol. 54 No.1,10August, 2013

[4] X. Hu, J. K. Wang, C. R. Wang, and C. Wang, -Is mobility always harmful to routing protocol performance of MANETs? $\|$ in Proc. Of International Conference on Cyber-Enabled Distributed Computing and Knowledge Discovery, pp. 108-112, 2010 .

[5] Y. Khamayseh, O. M. Darwish, and S. A. Wedian, -MA- AODV: Mobility Aware Routing Protocols for Mobile Ad hoc Networks, $\|$ in Proc. of Fourth International Conference on Systems and Networks Communications IEEE, pp. 25-29, 2009.

[6] W. Wang and C. Amza, -Motion-based Routing for Opportunistic Ad-hoc Networks, $\|$ in Proc. of 14th ACM international conference on Modeling, analysis and simulation of wireless and mobile systems October 31-November 4, 2011, pp. 169-178.

[7] R. Akbani, T. Korkmaz, and G .V. S. Raju, -HEAP: A packet authentication scheme for mobile ad hoc networks, $\|$ Ad Hoc Networks, vol. 6, no. 7, pp. 1134 1150, 2008.

[8] A. Boukerche et al., -Routing protocols in ad hoc networks: A survey,॥ Computer Networks: The International Journal of Computer and Telecommunications Networking, vol. 55, no. 13. pp. 3032-3080, May 2011.

[9] A. Boukerche, B. Turgut, N. Aydin, M. Z. Ahmad, L. Bölöni, and D.Turgut, - Routing protocols in ad hoc networks: A survey,\| -Elsevier Computer Networks", 55 (2011) 3032-3080.

[10] C. E. Perkins and P. Bhagwat, - Highly Dynamic Destination-Sequenced Distance-Vector (DSDV) for Mobile Computers,\| -Proc. ACM Conf. Communications Architectures and Protocols", London, UK, August 1994, pp.234-244.

[11] Piyush Chouhan, Ashish Mandloi and Ratnkant Jain-Route Selection in Mobile Ad Hoc Network On The Basis Of Routing Protocoll International Journal of Technology Research and Management ISSN (Online): 2348-9006 Vol 2 Issue 9 September 2015, pp.1-4. 